\title{
REDE DE ASSISTÊNCIA À SAÚDE DA CRIANÇA AUTISTA SOB A ÓTICA DAS MÃES
}

\author{
AUTISTIC CHILD HEALTH ASSISTANCE NETWORK UNDER THE OPTICS OF \\ MOTHERS
}

\author{
Ayrtton Anacleto Lima Dos Santos ${ }^{1}$ \\ Maria Emília Ferraz Almeida de Melo \\ Denyse Brito Nunes ${ }^{3}$
}

RESUMO: Objetivo: Avaliar a percepção das mães de crianças com autismo acerca da rede de cuidado integral a saúde dos seus filhos. Metodologia: Trata-se de um estudo descritivo, exploratório, de corte transversal, cuja amostra foi formada por mães de crianças com autismo atendidas na Associação de Pais e Amigos do Excepcionais (APAE) da cidade de Petrolina-PE. Foi entregue um questionário semi-estruturado às mães, contendo perguntas acerca de dados pessoais e informações de acesso aos serviços de saúde, que contemplavam avaliação, intervenção, acompanhamento no contexto domiciliar e educacional. Resultados: houve prevalência de mães que residem em cidades circunvizinhas, com dificuldades de transporte para chegar aos serviços de saúde e no agendamento de consultas nas Unidades Básicas de Saúde. Quanto ao acompanhamento de seu filho por profissionais de saúde, esse era realizado, principalmente, pelos neurologistas e fonoaudiólogos e em menor número por fisioterapeutas e nutricionistas. Conclusão: Foram detectadas algumas das fragilidades da assistência à saúde dessas crianças, como dificuldades de acesso aos serviços de saúde, bem como algumas potencialidades, evidenciadas pelo bom acolhimento dos profissionais que prestam assistência às famílias.

\footnotetext{
I Pós-graduado em Saúde do Trabalhador pela Faculdade Educamais. Graduado em Fisioterapia pela Universidade de Pernambuco (UPE). Fisioterapeuta com atuação na docência nas instituições: Centro Técnico Senhor do Bonfim (CETESB) e Complexo Educacional FESN para cursos de nível técnico. E-mail: ayrtton.anacleto@gmail.com.

${ }^{2}$ Doutorado em Educação em Ciências Química da Vida e Saúde Pela Universidade Federal de Santa MariaUFSM - Furg. Mestrado em Patologia- Universidade Federal de Pernambuco. Especialização em Especialização em Neuroeducação e Primeira Infância- Centro Iberoamericano de Neurociências, Educação e Desenvolvimento Humano. Especialização em Especialização Profissional em Fisioterapia NeurofuncionalAssociação Brasileira de Fisioterapia Neurofuncional- Especialização em Terapial Manual e Postural- Centro de Ensino Superior de Maringá. Graduação em Fisioterapia- Faculdade Integrada do Recife. Atualmente é professora adjunta do curso de Fisioterapia da Universidade de Pernambuco (UPE), nas disciplinas de Fisioterapia Neurofuncional, Fisioterapia aplicada à Saúde da Criança e do Adolescente e Estágio Supervisionado. Tem experiência na área de Fisioterapia, com ênfase em Fisioterapia Neurofuncional e Reeducação Postural Global (RPG), atuando principalmente nos seguintes temas: fisioterapia, neurologia, terapia manual e postural, RPG, Conceito Bobath (adulto e infantil), Facilitação Neuromuscular Proprioceptiva e Promoção à Saúde. É tutora no projeto PET Interprofissionalidade pela UPE campus Petrolina. Universidade Pernambuco.E-mail: emilia_ferraz@hotmail.com.

${ }^{3}$ Mestre em Ergonomia pela Universidade Federal de Pernambuco (UFPE). Especialização em Saúde Coletiva e Sociedade pelo Instituto Brasileiro de Pós-Graduação e Extensão. Graduação em Fisioterapia- Centro Universitário Maurício de Nassau - Recife. Docente da UNINASSAU e Faculdade São Francisco de Juazeiro (FASJ).E-mail: ft.denysebrito@hotmail.com.
} 
Palavras-chaves: Transtorno Autístico. Criança. Assistência Integral à Saúde. Sistema Único de Saúde.

ABSTRACT: Objective: To evaluate the perception of the mothers of children with autism about the integral health care network of their children. Methodology: This is a descriptive, exploratory, cross-sectional study whose sample was formed by mothers of children with autism treated at the Associação de Pais e Amigos do Excepcionais (APAE) of the city of Petrolina-PE. A semi-structured questionnaire was sent to the mothers,containing questions about personal data and information on access to health services, which included evaluation, intervention, follow-up in the home and educational context. Results: there was a high prevalence of mothers residing in surrounding cities, with transportation difficulties to reach the health services and in the scheduling of consultations in the Basic Health Units, was observed. Regarding the follow-up of their child by health professionals, this age performed mainly by neurologists and speech therapists, and in a smaller number by physiotherapists and nutritionists. Conclusion: Some of the weaknesses in the health care of these children were detected, such as difficulties in accessing health services, as well as some potentialities, evidenced by the good reception of professionals who provide assistance to families.

Keywords: Autistic Disorder. Child. Comprehensive Health Care. Unified Health System

\section{INTRODUÇÃO}

O Autismo Infantil foi definido por Kanner na década de 40, como uma condição com características comportamentais bastante específicas, de início precoce e incidência predominante no sexo masculino ${ }^{(\mathrm{I})}$. Asperger, em 1944, propôs a definição da psicopatia autística como um transtorno severo na interação social, uso pedante da fala, desajeitamento motor e incidência apenas no sexo masculino ${ }^{(2)}$. No entanto, os conceitos teóricos sobre a etiologia e dinâmica do mesmo vêm se modificando ao longo dos anos. $O$ autismo infantil é visto como um desenvolvimento anormal ou alterado, manifestado antes dos três anos de idade, apresentando perturbação do funcionamento nas áreas de interação social e comunicação, além de comportamento repetitivo (3).

A assistência integral à saúde dessas crianças parte do pressuposto de que há uma pluralidade de hipóteses etiológicas sem unanimidade conclusiva e de variedade de formas clínicas e/ou comorbidades, que podem acometer esses pacientes. Por isso, desde o processo de diagnóstico ao tratamento, é preciso o acompanhamento de uma equipe multiprofissional, sendo necessária uma avaliação com exames neurológicos, metabólicos e genéticos, bem como um tratamento que abrange atendimentos individuais, à família e atividades em grupo $^{(4)}$.

Após inúmeras audiências públicas, em 2012 foi aprovada a Lei 12.764, que dispõe sobre a Política Nacional de Proteção dos Direitos da Pessoa com Transtorno do Espectro do Autismo. Anos depois, o Decreto no 8.368, de dezembro de 2014, regulamentou essa lei, instituiu os direitos e obrigações para as pessoas com transtorno do espectro autista relacionados à saúde, como o direito de atendimento pelo Sistema Único de Saúde - SUS, além dos direitos à proteção e educação ${ }^{(5)}$.

No estado de Pernambuco, a lei estadual de no 47/2015 foi aprovada no ano de 2015 e prevê que a criança com autismo tenha direito a acompanhamento especializado, inclusão 
dos estudantes com o transtorno nas classes comuns de ensino público e privado e amplia a proteção à pessoa autista. No entanto, ao que parece, a rede de atenção integral à saúde dessas crianças ainda se encontra imatura e pouco estruturada ${ }^{(6)}$. As crianças com autismo assistidas pelo SUS de Petrolina são referenciadas para atendimento na Associação de Pais e Amigos dos excepcionais (APAE), devido a carência de profissionais e serviços para a assistência integral à saúde dessas crianças. Dessa forma, essa rede de atenção não se encontra bastante estruturada, a ponto de suprir a demanda dos casos do município.

Devido à falta de estudos voltados para um maior conhecimento e estruturação da rede de atenção à saúde integral à criança autista, o que é ainda mais raro em regiões do interior do Nordeste, faz-se necessário que se conheça os principais obstáculos enfrentados pelas famílias dessas crianças na busca pelos cuidados a saúde dos seus filhos. De posse dessas informações, possibilita-se ao governo, e também à iniciativa privada, uma melhor estruturação para atender às necessidades de atenção às crianças autistas. Esse trabalho tem como objetivo avaliar a percepção das mães de crianças com autismo acerca da rede de cuidado integral a saúde dos seus filhos, atendidos por uma instituição filantrópica da cidade de Petrolina-PE.

\section{METODOLOGIA}

Tratou-se de um estudo descritivo, exploratório, de corte transversal, desenvolvido na APAE de Petrolina-PE, nos meses de outubro a novembro de 2017. Esta é uma Organização Social, cujo objetivo principal é promover a atenção integral à pessoa com deficiência intelectual e múltipla, prestando serviços de educação, saúde e assistência social. A instituição conta com 40 crianças cadastradas com autismo, atendidas uma vez por semana no setor de reabilitação, além de estudar na própria instituição.

A amostra do estudo foi intencional e censitária, com todas as mães de crianças com diagnóstico de autismo ou espectro autista, que estavam cadastrados na APAE e que aceitaram participar do estudo. Foram excluídas 9 mães de crianças que não responderam ao questionário após cinco tentativas de contato, realizadas em dias diferentes.

Foi utilizado um questionário auto referido, contendo informações sociodemográficas, ocupacionais e de acesso aos serviços de saúde. O questionário foi constituído pelas dados pessoais e informações de acesso aos serviços de saúde, que contemplavam avaliação, intervenção, acompanhamento no contexto domiciliar e educacional. As questões foram objetivas, de múltipla escolha, com alternativas de "sim", "não" e "não sei", bem como utilização de Escala de Likert (1932), onde constavam alternativas como: "satisfeito", "pouco satisfeito", "nem satisfeito nem insatisfeito", "pouco insatisfeito", "muito insatisfeito", tornando-se assim de fácil preenchimento e compreensão. O questionário foi construído pela equipe de pesquisa baseada no trabalho de Shirley e Munhoz (2013)(7).

Foram realizadas cinco reuniões com as mães das crianças no auditório existente na APAE, onde foi exposto a elas o tema da pesquisa, assim como os objetivos do estudo, de forma clara para o entendimento de todas. Posteriormente, o entrevistador aplicou o questionário e informou as mães a respeito de uma nova reunião, onde serão apresentados os resultados obtidos, e, consequentemente, trazendo informações a respeito das redes de atenção que existem no município e em cidades próximas.

As informações foram analisadas de froma descritiva e utilizou-se frequências absoluta e relativa para as variáveis categóricas e medidas de tendência central (média) e de dispersão 
(desvio padrão) para as variáveis numéricas. O processamento e a análise dos dados, bem como a apresentação destes através de tabelas e gráficos foi realizado por meio do programa Microsoft Excel versão 2010.

As mães das crianças com autismo que participaram do inquérito foram informadas sobre o objetivo da pesquisa e assinaram o Termo de Consentimento Livre e Esclarecido (TCLE), sendo garantido o sigilo e anonimato das informações. O trabalho foi submetido e aprovado pelo comitê de Ética em Pesquisa da Universidade de Pernambuco sob Parecer № 2.361.678, de acordo com as exigências da Res. no. 466/2012 CNS/CONEP.

\section{RESULTADOS}

Foram entrevistadas 31 mães de crianças autistas, cuja idade variou entre 24 e 55 anos, com uma média de $36,8( \pm 7,86)$ anos. Dessas, observa-se a grande prevalência de mães que residem em cidades circunvizinhas, num total de 18 (58,8\%), seguido de II mães $(35,48 \%)$ residentes em Petrolina. No que diz respeito aos aspectos sociodemográficos, verificou-se que, em sua maioria (38,70\%) são solteiras, com Ensino Médio Completo (35,48\%), tem renda familiar de I salário-mínimo $(48,38 \%)$ e com número de filhos variando entre i e 5 , tendo como média 2,26 filhos (Tabela I).

Tabela I: Distribuição das mães entrevistadas com relação aos fatores sociodemográficos (Petrolina/2017).

\begin{tabular}{lcc}
\hline VARIÁVEL & N & $\%$ \\
\hline IDADE (Média) & 36,8 & \\
MUNICÍPIO DE RESIDÊNCIA & & \\
Petrolina & II & 35,48 \\
Juazeiro & 2 & 6,45 \\
Outros & I8 & 58,06 \\
ESTADO CIVIL & & \\
Solteira & I2 & 38,70 \\
Casada & IO & 32,25 \\
Outros & 9 & 29,03 \\
NIVEL DE ESCOLARIDADE & & \\
Analfabeto/Fundamental Incompleto & 8 & 25,8 \\
Fundamental completo & 8 & 25,8 \\
Médio Completo & II & 35,48 \\
Superior Completo & 4 & 12,90 \\
NÚMERO DE FILHOS (Média) & 2,26 & \\
RENDA FAMILIAR & & \\
Sem renda & 6 & 19,35 \\
Até I salário mínimos & I5 & 48,38 \\
I a 3 salários-mínimos & 8 & 25,8 \\
3 ou mais salários mínimos & 2 & 6,45 \\
\hline N=Número; \%percentual & &
\end{tabular}

$\mathrm{N}=$ Número; \%=percentual
Fonte: questionário do pesquisador

As percepções sobre o acesso aos serviços de saúde, dificuldade de agendamento e o tempo gasto para locomoção entre residência e os serviços oferecidos estão expostos na 
Tabela II. Observou-se que i6 mães (51,61\%) relataram ter dificuldade de transporte para chegar à unidade básica de saúde. Já a dificuldade sobre transporte para aos demais serviços foi referida por 19 delas $(6 \mathrm{I}, 29 \%)$. O tempo médio gasto para o acesso à Unidades Básicas de Saúde (UBS) é de 45,2 minutos e, aos demais serviços de saúde, de 81,53 minutos.

Quanto ao agendamento de consulta nas UBS 22 mães (70,96\%) afirmaram ter dificuldades no agendamento. Quando questionadas se eram assistidas por algum profissional de saúde em domicílio, apenas 9 delas (29,03\%) disseram ser assistidas, sendo que estas relataram ser assistidas apenas por agentes de saúde (Tabela II).

Tabela II: Dificuldades encontradas pelas mães para utilização dos serviços de saúde (Petrolina/2017).

\begin{tabular}{lccccc} 
VARIÁVEL & \multicolumn{2}{c}{ SIM } & \multicolumn{2}{c}{ NÃO } \\
& N & \% & N & \% \\
\hline Dificuldade de transporte para a UBS & I6 & 51,61 & I5 & 48,39 \\
Dificuldade de transporte para os serviços de saúde & I9 & 61,29 & II & 35,48 \\
Dificuldade de agendamento de consulta & 22 & 70,96 & 9 & 29,03 \\
Assistência por profissional de saúde em casa & 9 & 29,03 & 22 & 70,96 \\
Tempo de deslocamento para a UBS (Média) & \multicolumn{2}{c}{45,2} \\
Tempo de deslocamento para Serviços (Média) & \multicolumn{2}{c}{81,53} & & \\
\hline Fonte: questionário do pesquisador & \multicolumn{5}{c}{}
\end{tabular}

As mães referiram, ainda, sobre os profissionais que acompanhavam seus filhos atualmente, que este acompanhamento era realizado, principalmente, pelos neurologistas $(\mathrm{N}=22,70,96 \%)$ e fonoaudiólogos $(\mathrm{N}=23,74,19 \%)$, como visto na Figura I. Os profissionais que menos assistiam essas crianças foram o fisioterapeuta e o nutricionista, sendo referido por apenas 3 mães $(9,67 \%)$. Apenas uma mãe $(3,25 \%)$ afirmou que seu filho é atendido por outro profissional de saúde, que, neste caso, era o médico clínico geral (Figura I).

Figura r: Distribuição dos profissionais que acompanham as crianças autistas (Petrolina/2017).

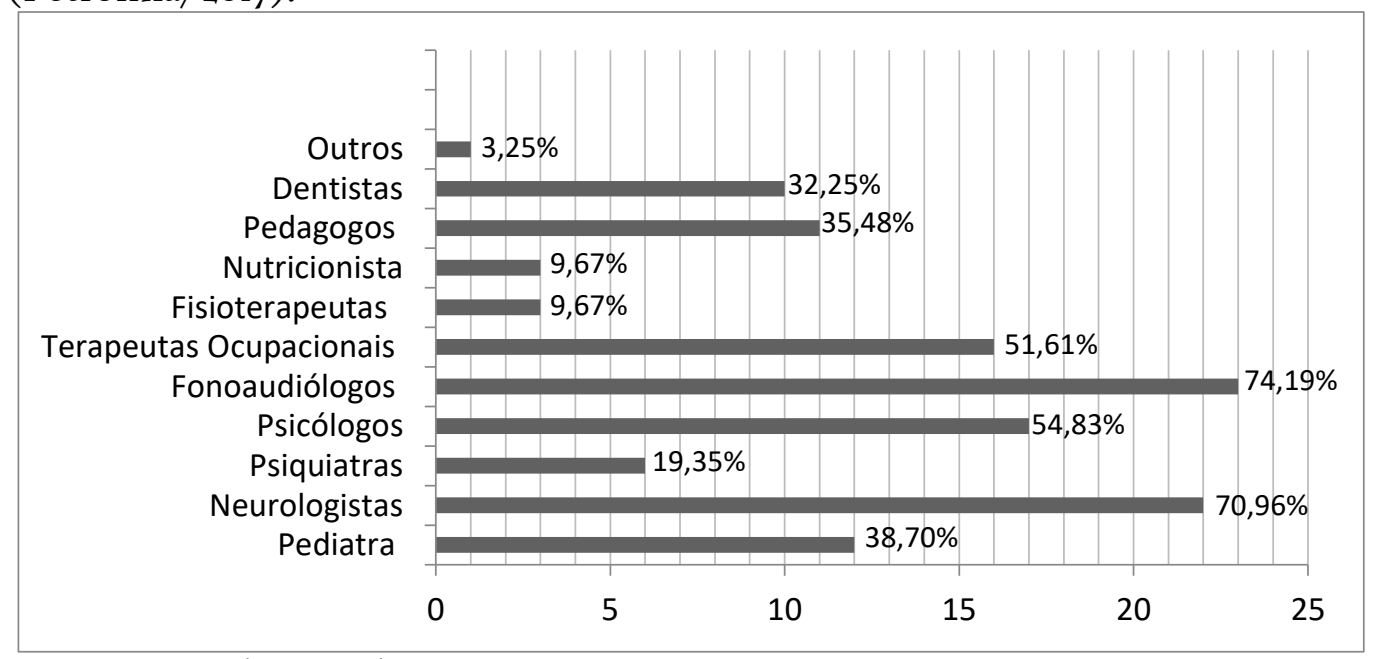

Fonte: questionário do pesquisador

Já em relação à quantidade de vezes que essas crianças são atendidas, foi verificado que, por ano, apenas 2 crianças $(6,45 \%)$ não fazem nenhum atendimento médico. Outras 23 crianças $(74,19 \%)$ vão de I a I2 vezes por ano a uma consulta médica. No que se refere aos 
atendimentos dos demais profissionais de saúde e realização de exames (Tabela III), foi observado que 28 crianças (90,32\%) não vão de maneira alguma ao serviço de fisioterapia. $\mathrm{Na}$ área de reabilitação, predominam as idas ao fonoaudiólogo, referida por 20 mães $(64,51 \%)$, e ao Terapeuta Ocupacional, de acordo com 17 delas $(54,83 \%)$, indo mais de 2 vezes por mês.

Quando questionadas sobre os exames realizados em seus filhos, 3 delas (9,67\%) relataram que seus filhos nunca fizeram nenhum tipo de exame, sendo que, dentro os exames mais realizados, estão os laboratoriais (8o,64\%), seguido pela avaliação da audição $(45,6 \%)$ (Tabela III).

Tabela III: Quantidade de consultas realizada mensalmente e exames feitos pelas crianças atualmente (Petrolina/ 2017).

\begin{tabular}{|c|c|c|c|c|c|c|}
\hline \multirow[t]{2}{*}{ VARIÁVEL } & \multicolumn{2}{|c|}{ NÃO VAI } & \multicolumn{2}{|c|}{$\begin{array}{c}\text { ATE IX POR } \\
\text { MÊS }\end{array}$} & \multicolumn{2}{|c|}{$\begin{array}{l}\text { MAIS DE } 2 \mathrm{X} \\
\text { POR MÊS }\end{array}$} \\
\hline & $\mathbf{N}$ & $\%$ & $\mathbf{N}$ & $\%$ & $\mathbf{N}$ & $\%$ \\
\hline FISIOTERAPIA & 28 & 90,32 & I & 3,25 & 2 & 6,45 \\
\hline FONOAUDIÓLOGO & Io & 32,25 & I & 3,25 & 20 & 64,51 \\
\hline $\begin{array}{l}\text { TERAPEUTAS } \\
\text { OCUPACIONAIS }\end{array}$ & I4 & 45,16 & o & o & 17 & 54,83 \\
\hline EXAMES & $\mathbf{N}$ & $\%$ & & & & \\
\hline Nenhum & 3 & 9,67 & & & & \\
\hline Laboratoriais & 25 & 80,64 & & & & \\
\hline Cariótipo & 2 & 6,45 & & & & \\
\hline Cardiológicos & I & 3,25 & & & & \\
\hline Raio- $\mathrm{x}$ & 6 & 19,35 & & & & \\
\hline Avaliação da visão & Io & 32,25 & & & & \\
\hline Avaliação da audição & $\mathrm{I} 4$ & 45,16 & & & & \\
\hline $\begin{array}{l}\text { Outros tipos de } \\
\text { exames }\end{array}$ & 7 & 22,58 & & & & \\
\hline
\end{tabular}

No tocante à relação usuário (mãe e criança) com o profissional de saúde, foi relatado por estes que não há dificuldade no acolhimento, onde, por unanimidade, as 3I mães (Ioo\%), afirmaram sentirem-se acolhidas pelos profissionais de saúde. Nesse sentido, 23 delas (74,19\%) afirmaram que existe vínculo entre os profissionais e sua família, número que se repetiu quando questionadas se foram orientadas quanto à estimulação precoce dos seus filhos. Já no quesito relacionado ao fato de os profissionais que atendiam seu filho (a) se conhecer e discutir o caso do mesmo, apenas is (48,39\%) afirmaram que essa prática acontecia.

O grau de satisfação dessas mães em relação a tempo para marcação das consultas, espaço onde são realizados atendimentos, profissionais e orientação quanto aos direitos do seu filho(a) estão expostos na Tabela IV. Observou-se que $35,48 \%$ das mães estão muito insatisfeitas em relação à demora no tempo de espera para as consultas marcadas na UBS, apesar de sentirem-se muito satisfeitas com o espaço físico onde são atendidas $(38,70 \%)$. Com relação aos profissionais que assistem a criança, observou-se muita satisfação por parte de 25 mães $(80,64 \%)$, bem como muita satisfação com a orientação recebida acerca dos direitos das crianças autistas $(61,29 \%)$. 
Tabela IV: Grau de satisfação das mães com os serviços e profissionais de saúde e orientação dada sobre os direitos das crianças autistas (Petrolina/2017).

\begin{tabular}{|c|c|c|c|c|c|c|c|c|c|c|}
\hline \multirow[t]{2}{*}{ VARIAVEL } & \multicolumn{2}{|c|}{$\begin{array}{c}\text { Muito } \\
\text { Satisfeito }\end{array}$} & \multicolumn{2}{|c|}{$\begin{array}{c}\text { Pouco } \\
\text { satisfeito }\end{array}$} & \multicolumn{2}{|c|}{$\begin{array}{l}\text { Nem Satisfeito, } \\
\text { nem insatisfeito }\end{array}$} & \multicolumn{2}{|c|}{$\begin{array}{c}\text { Pouco } \\
\text { insatisfeito }\end{array}$} & \multicolumn{2}{|c|}{$\begin{array}{c}\text { Muito } \\
\text { insatisfeito }\end{array}$} \\
\hline & $\mathbf{N}$ & $\%$ & $\mathbf{N}$ & $\%$ & $\mathbf{N}$ & $\%$ & $\mathbf{N}$ & $\%$ & $\mathbf{N}$ & $\%$ \\
\hline $\begin{array}{l}\text { Tempo de } \\
\text { consultas }\end{array}$ & 3 & 9,67 & 7 & 22,58 & 7 & 22,58 & 3 & 9,67 & II & 35,48 \\
\hline $\begin{array}{l}\text { Espaço de } \\
\text { atendimentos }\end{array}$ & 12 & 38,70 & II & 35,48 & 4 & 12,90 & 3 & 9,67 & I & 3,25 \\
\hline $\begin{array}{l}\text { Qualidade dos } \\
\text { Profissionais }\end{array}$ & 25 & $\begin{array}{c}80,6 \\
4\end{array}$ & I & 3,25 & 2 & 6,45 & 2 & 6,45 & I & 3,25 \\
\hline $\begin{array}{l}\text { Orientação } \\
\text { recebida quanto } \\
\text { aos direitos }\end{array}$ & 19 & 61,29 & 7 & 22,58 & 3 & 9,67 & I & 3,25 & I & 3,25 \\
\hline
\end{tabular}

\section{DISCUSSÃO}

As redes de assistência à Saúde se conceituam na integralidade do sujeito e na disposição de uma rede de cuidados que se ajuste em atender integralmente à diversidade das demandas ${ }^{4}$. Pode-se destacar, então, que somente através de uma organização em rede, e não apenas um serviço ou equipamento, se é possível fazer um atendimento pautado na complexidade das demandas, principalmente daquelas que necessitam de inclusão e são secularmente estigmatizadas ${ }^{(8)}$.

No âmbito da acessibilidade geográfica, a maioria das mães reside em outros municípios, o que pode ser um fator de dificuldade no acesso aos serviços especializados para seus filhos. Sabe-se que as barreiras relacionadas à distância estão associadas ao nível de complexidade dos serviços: quanto menos especializados, mais próximos estão da população(9).

Mesmo a área do estudo sendo urbana, percebeu-se que e a mesma não é atendida por um sistema de transporte eficiente, visto que algumas mães possuíam mais facilidades do que outras no que diz respeito ao acesso aos transportes. Nesse sentido, as UBS correspondem ao conceito de acessibilidade geográfica com maior facilidade ${ }^{(\text {io) }}$. O tempo e as dificuldades com locomoção vistas neste estudo foram menores para as UBS se comparado ao dos demais serviços de saúde, visto que as unidades de saúde estão localizadas no território onde as famílias a elas cadastradas residem.

Por outro lado, é importante ressaltar que a existência de serviço em determinado local, apesar de constituir um aspecto fundamental para garantir acesso, não garante a sua efetiva utilização ${ }^{\text {II. }}$ A distância dos serviços às residências dos usuários pode ser contrabalanceada desde a facilitação de transporte, a presença de um sistema de referência aos serviços hospitalares e mecanismos de acolhimento que facilitem a obtenção de cuidado $(12,12)$.

Nota-se na presente pesquisa que os resultados quanto à renda salarial chamam atenção para as possíveis condições precárias de vida dessas mães, que em sua maioria são solteiras, pouco escolarizadas e com mais de um filho. Estudos têm revelado que pessoas inseridas nos grupos de renda mais baixa procuram menos os serviços de saúde ou 
apresentam menor probabilidade de usá-los ${ }^{(13)}$. Isso reduz o acesso dessas mães aos serviços de saúde, assim como a oportunidade dessas de levar seus filhos para fazer exames que muitas vezes não são oferecidos pelo sistema único de saúde ${ }^{(\mathrm{I} 4)}$.

Além disso, o acesso envolve as necessidades e predisposição do indivíduo para utilização do serviço de saúde, por condições que permitam ou impeçam a sua utilização, muitas vezes determinados por situações de vulnerabilidade da população ${ }^{(14,15)}$. Extrapolando a simples dimensão geográfica, sendo dessa forma algo multidimensional, levando em conta aspectos políticos, econômico social (renda, seguro de saúde), técnica, organizacional (disponibilidade de serviços, distância e tempo de espera) e simbólica ${ }^{\left({ }^{15-17}\right)}$.

O tratamento para as crianças com autismo tem como finalidade trabalhar as habilidades de interação social e linguagem (18). Sendo a linguagem um dos tripés que sustenta o diagnóstico autístico, a mesma é uma das preocupações iniciais dos pais no decorrer do desenvolvimento da criança. $\mathrm{Na}$ pesquisa observou-se que, apesar de não serem todas as crianças atendidas pelo profissional fonoaudiólogo, ou nem todas serem atendidas semanalmente, quando perguntado às mães sobre quais os profissionais acompanhavam estas atualmente, o maior índice foi de atendimento com fonoaudiólogos. Este tipo de tratamento é um bom indicativo do prognóstico, visto que os prejuízos na linguagem estão diretamente associados ao baixo nível de cognição e a comportamentos disruptivos (ig).

Quando uma criança é diagnosticada com autismo, o profissional responsável deve encaminhar a mesma para um tratamento que inclui uma equipe multidisciplinar, composta por terapeutas ocupacionais, fisioterapeutas, fonoaudiólogos, neurologistas, profissionais que lidam com problemas alimentares $\mathrm{e} / \mathrm{ou}$ de sono ${ }^{(20)}$. No entanto, os resultados da atual pesquisa mostram a fragilidade dessa assistência multiprofissional na amostra estudada, haja vista os índices de atendimentos com fisioterapeutas e nutricionistas aquém do ideal.

Em relação à fisioterapia, que foi referido por apenas 3 mães, pode refletir a fragilidade do serviço no que diz respeito à carência de vagas para suprir a demanda de atendimentos e na falta de apoio financeiro para ampliação do serviço. Visto que as crianças com autismo possuem déficits que comprometem a interação social, comunicação e flexibilidade no raciocínio, podendo apresentar, também, comprometimentos motores, a fisioterapia se $\mathrm{faz}$ necessária a intervenção precoce ${ }^{(2 \mathrm{r})}$. Esta utiliza a neuroplasticidade cerebral para auxiliar no desenvolvimento motor, ativando áreas de concentração, integração social, melhorando a sua qualidade de vida ${ }^{(22)}$.

$\mathrm{Na}$ pesquisa pode-se observar, também, que apenas uma minoria era assistida por algum profissional de saúde em domicílio, e esses eram agentes de saúde ${ }^{(23)}$. A assistência domiciliar vem como um facilitador ao acesso precoce aos serviços de saúde, levando condições de atendimento e recursos para a melhor evolução do seu quadro clínico. Assim, esta é uma ferramenta estratégica de diminuição de despesas para os serviços de saúde, manutenção da qualidade de saúde e mais humanização( ${ }^{(24)}$.

Apesar das dificuldades encontradas na estruturação e acesso à rede de assistência a essas crianças, percebeu-se uma boa satisfação das mães com relação aos profissionais, desde o acolhimento ao vínculo com as famílias. $\mathrm{O}$ acolhimento é uma das principais diretrizes da Política Nacional de Humanização (PNH). Ele vem garantir ao usuário o direito ao acesso universal, sem exclusão social e com equidade. Sabe-se que o serviço de saúde, hoje, é direcionado para o problema do usuário, para sua doença e não para o paciente no seu contexto singular. Com o acolhimento assim, os atendimentos realizados se direcionam para 
o usuário e não para a sua doença, além de mudar o foco da atenção, que antes era apenas do médico e passa para o foco de uma equipe multiprofissional ${ }^{(25)}$.

\section{CONCLUSÃO}

O desenvolvimento do presente estudo possibilitou uma análise preliminar no que diz respeito à percepção das mães de crianças autistas acerca do cuidado integral à saúde dos filhos, assistidos pela APAE de Petrolina, onde foram observadas as fragilidades dessas mães, como dificuldades de acesso (residências em outros municípios da região, o longo tempo para chegada aos serviços de saúde e dificuldade de transporte) e acessibilidade (demora em marcação de consultas, poucos profissionais que acompanham as crianças, pequena quantidade de crianças que são atendidas por fisioterapeutas) aos serviços de saúde. Mas também potencialidades, como o bom acolhimento dos profissionais que atendem essas crianças, assim como o vínculo e as orientações dadas a essas famílias. Dada a importância dessa assistência integral, torna-se necessário o desenvolvimento de novas pesquisas, com maior número de mães e avaliando aspectos qualitativos não abordados neste estudo. Assim como se faz necessária a criação de um centro de referencia na cidade de Petrolina-Pe para atender na integralidade essas criança e políticas publicas que garantam todos os seus direitos.

\section{REFERENCIAS:}

I. Kanner L. Autistic disturbances of affective contact. Nervous Child. 1943;2:217-50.

2. Asperger H. Die autistischen psychopathen in kindesalter. Arch Psychiatr Nervenkr. 1944;110:76-ıз6.

3. Organização Mundial da Saúde. Classificação estatística internacional de doenças e problemas relacionados à saúde: CID ıo. ıo a. ed. São Paulo: Edusp; 1998.

4. Ministério da Saúde (Brasil). Secretaria de Atenção à Saúde. Departamento de Atenção Especializada e Temática. Linha de Cuidado para a atenção às pessoas com transtornos do espectro do autismo e suas famílias: $\mathrm{Na}$ rede de atenção psicossocial do sistema único de saúde. Ministério da Saúde, Secretaria de Atenção à Saúde, Departamento de Atenção Especializada e Temática. - Brasília: Ministério da Saúde, 2015. 156 p.il.. Acesso em: I2 Set. 2017. Disponível em: 〈http:// editora.saude.gov.br >

5. Brasil. Lei 12764, de 27 Dez. de 2012, Institui a Política Nacional de Proteção dos Direitos da Pessoa com Transtorno do Espectro Autista. Disponível em: 〈http://www.planalto.gov.br/ccivil_03/_ato20II-2014/2012/lei/li2764.htm.> Acesso em: 27/07/2017.

6. Brasil. Lei 47, de 27 abril de 2015, Institui uma Legislação específica no Estado de Pernambuco sobre a proteção e os direitos da pessoa com Transtorno de Espectro Autista Disponível em: <http://www.alepe.pe.gov.br/proposicao-textocompleto/?numero $=47 / 2015 \&$ docid $=>$ Acesso em: $16 / 08 / 2017$.

7. Modes PSSA, Gaiva MAM. Satisfação das usuárias quanto à atenção prestada à criança pela rede básica de saúde. Esc. Anna Nery [Internet]. 2013 Aug [cited 2017 Dec 03] ; $\quad{ }_{17}(2)$ ) $\quad 455-465 . \quad$ Available from: http://www.scielo.br/scielo.php?script=sci_arttext\&pid $=S_{1414}-$

81452013000300455\&lng=en. http://dx.doi.org/ro.1590/SI4I4-81452013000300008. 
8. Ministério da Saúde (Brasil). Secretaria de Atenção à Saúde. DAPE. Coordenação Geral de Saúde Mental. Reforma psiquiátrica e política de saúde mental no Brasil. Documento apresentado à Conferência Regional de Reforma dos Serviços de Saúde Mental: 15 anos depois de Caracas. OPAS. Brasília, novembro de 2005.

9. Albuquerque MSV, Lyra TM, Farias SF, Mendes MFM, Martelli PJL. Acessibilidade aos serviços de saúde: uma análise a partir da Atenção Básica em Pernambuco. Saúde debate [Internet]. 2014 Oct [cited 2017 Dec 03] ; 38( spe ): 182-194. Available from: http://www.scielo.br/scielo.php?script=sci_arttext\&pid=Soro3II04201400060or82\&lng=en. http://dx.doi.org/ro.5935/o103-1104.2014Sor4.

Io. Donabedian A. An introduction to quality assurance in health care. New York: Oxford University, 2003.

II. Travassos C, Viacava F. Acesso e uso de serviços de saúde em idosos residentes em áreas rurais, Brasil, 1998 e 2003. Cadernos de Saúde Pública, Rio de Janeiro. 23, io, 24902502, 2007.

I2. Pinheiro RS. et al. Gênero, morbidade, acesso e utilização de serviços de saúde no Brasil. Ciência \& Saúde Coletiva, Rio de Janeiro.7,4, 687- 707, 2002.

13. Dias CJS. Utilização de serviços ambulatoriais de saúde em Pelotas, Rio Grande do Sul, Brasil: alguns fatores relacionados com as consultas médicas acima da média. Cad Saude Publica 2008; 24(2):353-363

I4. Obrist B. et al. Access to health care in contexts of livelihood insecurity: a framework for analysis and action. Plos Medicine, Cambridge.4, I0, 1584-1588, 2007.

15. Jesus WLA, Assis MMA. Revisão sistemática sobre o conceito de acesso nos serviços de saúde: contribuições do planejamento. Cienc Saúde Coletiva, Rio de Janeiro. 15, I, I6II70, 2010.

16. Assis MMA, Jesus WLA. Acesso aos serviços de saúde: abordagens, conceitos, políticas e modelo de análise. Ciênc Saúde Coletiva, Rio de Janeiro.17, II, 2865-2875, 2012.

17. Souza ECFD et al. Acesso e acolhimento na atenção básica: uma análise da percepção dos usuários e profissionais de saúde. Cadernos de Saúde Pública, Rio de Janeiro. 24, I, IooIIO, 2008.

18. Nikolov R, Jonker J, Scahill L. Autismo: tratamentos psicofarmacológicos e áreas de interesse para desenvolvimentos futuros. Rev Bras Psiquiatr. 2006; 28(supl.I):s39-s46

19. Silva RA, Lopes HAS, Vitto LPM. Distúrbio de linguagem como parte de um transtorno global do desenvolvimento: descrição de um processo terapêutico fonoaudiológico. Rev Soc Bras Fonoaudiol. 2007;12(4):322-8.

20. Silva M, Mulick AJ. Diagnosticando o Transtorno Autista: Aspectos Fundamentais e Considerações Práticas. Revista Psicologia Ciência e Profissão, v.29, p.II6-I3I, 2009.

21. Cazorla GJJ; Cornellá ICJ. Las posibilidades de la fisioterapia en el tratamiento multidisciplinar del autismo. Pediatría atención primaria. v.i6, p.37-46, 2014.

22. Segura DC, Nascimento FC, Klein D. Estudo do Conhecimento Clínico dos Profissionais da Fisioterapia no Trabalho de Crianças Autistas. Arquivos de Ciência da Saúde Unipar, v.15, p.159-165, 2011.

23. Secretaria Municipal Da Saúde De Porto Alegre . Coordenadoria Geral de Vigilância da Saúde. A Atenção à Saúde da criança de zero a cinco anos deidadeDisponívelem: $<$ http://lproweb.procempa.com.br/pmpa/prefpoa/cgvs/usu_doc/e v_pub_2004_protocolooa5.pdf $>$. Acesso em: 05 dez. 2017. 
24. Raffa GC, Hernan CPL.A Importância do Home Care no Atual Cenário da Saúde.Disponível em: 〈http://www.saocamilo.br/jornalsaocamilo_online/sp/sp_pontodevistar8.htm〉. Acesso em: 05 dez. 2017.

25. Lopes FM. Acolhimento: uma revisão de literatura. Trabalho de conclusão de curso. Virgolânisa-MG: Universidade De Minas Gerais; 2009. 